\title{
IMAGING PERSPECTIVE OF PRIMARY EOSINOPHILIC ENTEROCOLITIS - A RARE CASE: REPORT
}

Nagarajaiah Y. N1, Prakash Jain R. B², Nitash Prakash³

\section{HOW TO CITE THIS ARTICLE:}

Nagarajaiah Y. N, Prakash Jain R. B, Nitash Prakash. "Imaging Perspective of Primary Eosinophilic Enterocolitis: A Rare Case: Report". Journal of Evolution of Medical and Dental Sciences 2014; Vol. 3, Issue 30, July 28;

Page: 8443-8449, DOI: $10.14260 /$ jemds/2014/3067

\begin{abstract}
A 28-year-old male patient presented with chronic pain abdomen in the right iliac fossa, intermittent vomiting and frequent defecation. Trans-abdominal ultrasonography and CT abdomen pelvis revealed long segment diffuse circumferential wall thickening of distal ileum, caecum and adjacent ascending colon. Colonoscopy revealed ulcers in the ileo-caecal valve with terminal ileal stricture. Blood eosinophilia, tuberculosis and parasitic infections were excluded. A diagnosis of enterocolitis was suspected. Brush biopsy could confirm the rare diagnosis of an eosinophilic enterocolitis with predominantly mucosal and sub-mucosal infiltration. The patient was started on corticosteroids. Six months later the patient relapsed with symptoms and signs of intestinal obstruction. Repeat ultrasonography showed the persistent bowel wall thickening. Colonoscopy showed stenosed and deformed ileo-caecal valve with chronic ulceration. Diagnosing Primary Enterocolitis is based on colonic biopsies, which is especially challenging in the absence of diagnostic criteria. It is necessary to eliminate secondary causes by ultrasonography/ CT abdomen pelvis. CT/MR imaging provides valuable information for diagnosis, guiding treatment and assessing response to therapy.
\end{abstract}

KEYWORDS: Eosinophilic entero-colitis, bowel wall thickening, bowel obstruction.

INTRODUCTION: Here is a case report of a patient with long standing gastrointestinal symptoms diagnosed as eosinophilic enteritis. The importance of this report is the rarity of the case and the symptoms and imaging findings mimics other chronic diseases affecting the bowel like tuberculosis and malignancy which are quiet common and it is important to consider this diagnosis as the treatment varies.

Eosinophilic gastroenteritis is a rare disease commonly affected are the stomach and small intestine, rarely sparing these it exclusively affects the small and large bowel. ${ }^{1}$ It can be either primary (idiopathic) or secondary as a result of systemic conditions such as hypereosinophilic syndrome, celiac disease, Crohn disease, Churg-Strauss syndrome, and helminthic infection or of drugs such as enalapril, gemfibrozil, cyclosporine, naproxen, clozapine, rifampicin and gold.2,3

Primary eosinophilic gastrointestinal disorders can be subgrouped as eosinophilic esophagitis (EE), eosinophilic gastroenteritis (EG), and eosinophilic colitis (EC) and allergic procto colitis of infancy. ${ }^{3}$ The imaging features of eosinophilic gastroenteritis depend on the site of involvement and the involved layer of the gastrointestinal tract (i.e., mucosa, muscle, or serosa). ${ }^{2}$

Corticosteroids are the mainstay of therapy and have good response rates, but patients often relapse after cessation of therapy. ${ }^{2}$

CASE REPORT: A 28-year-old male patient presented with chronic pain abdomen in the right iliac fossa and intermittent vomiting since 18 months. The patient complained of frequent defecation, 
about 4-5 times a day predominantly after taking food. No history of blood/mucus stained stools. No history of fever, loss of appetite, allergy. Past history was clinically insignificant.

Laboratory investigations revealed normal cytology with normal leukocyte count and eosinophil count. ESR was within normal limits. Monteux test and PCR for tuberculosis were negative. Stool examination ruled out parasitic infection. ELISA test for HIV was negative.

Abdominal radiograph and Barium meal follow through study was done and was normal. Terminal ileum and caecum were normal in position and ileo-caecal angle was normal.

Transabdominal Ultrasonography revealed long segment diffuse circumferential wall thickening of distal ileum, caecum and adjacent ascending colon. The wall thickness measured up to 8 $\mathrm{mm}$. Minimal pericaecal fluid was seen. No pathologically significant lymphadenopathy by standard size criteria (Fig. 1, 2 and 3). These findings were confirmed by contrast enhanced CT studies which revealed the same (Fig. 4 and 5).

Colonoscopy revealed few small ulcers in the caecum and linear ulceration in the ileo-caecal valve. The biopsy was performed and sent for histopathological examination.

Histopathological examination reveals focal ulceration of the mucosa. The (edematous) lamina propria shows moderately dense inflammatory cell infiltrate comprising of lymphocytes, plasma cells and eosinophils. No evidence of granulomatous or malignant cell features. The histopathological examination favored eosinophilic enterocolitis (Fig. 6).

The clinical findings, laboratory findings, imaging features, colonoscopy results and histopathological findings were correlated and a diagnosis of eosinophilic enterocolitis was made. The patient was treated with corticosteroids. Patient responded to the treatment and the steroid dose was gradually tapered as the symptoms reduced. Review ultrasound examination showed decrease in the bowel wall thickening.

After six months patient came to the hospital again presenting with vomiting and pain abdomen. The erect abdomen radiograph showed large air fluid levels in the central abdomen suggesting small intestinal obstruction. A repeat transabdominal ultrasound showed persistent bowel wall thickening. Barium meal follow through examination showed stricture at the terminal ileum with proximal small bowel loop dilation (Fig. 7).

Colonoscopy was done which showed stenosed, deformed ileo-caecal valve with chronic ulceration. The ileo-caecal valve was dilated with Controlled Radial Expansion balloon and biopsy was taken. The histopathology examination of the material revealed mucosal ulceration with mixed inflammatory cells comprising of polymorphs, lymphoid cells, few histiocytes and plenty of eosinophilic infiltration in the lamina propria. The corticosteroid administration was continued. A month later patient was asymptomatic, transabdominal ultrasound showed thickened bowel wall only in the terminal ileum, with normal/reduced wall thickness in the ileo-caecal valve, caecum and ascending colon.

DISCUSSION: Eosinophilic enteritis is a rare disorder of unknown etiology first reported in the year 1937 by Kaijser. ${ }^{4}$ Eosinophilic gastroenteritis may occur at any age, but it is most common in the 3rd and 4th decades, and there is a slight male predilection (male-to-female ratio, 1.4:1). Eosinophilic gastroenteritis is extremely rare, with an estimated prevalence of one in 100, 000 people. As many as $75 \%$ of patients have a history of Atopy and allergy, and peripheral eosinophilia is present in $75 \%-$ $100 \%$ of patients. ${ }^{2}$ 
Primary EC, an emerging disorder within the primary EGIDs, although distinctly uncommon, has become better defined over the past decade owing to the recent advances in imaging techniques, endoscopy and transmural biopsy. ${ }^{3}$ Diagnostic methods and high index of suspicion is needed in identifying and diagnosing the disease.

It can be classified with respect to the site of involvement (esophageal, gastric, enteric or colonic) or location of involvement (mucosal, muscular, serosal or diffuse).

The disorder is categorized as primary and secondary. EC in its primary form can be associated with other atopic conditions. Colonic eosinophilia can also occur secondary to helminthic infections (e.g. pin worms, hookworms), inflammatory bowel disease, autoimmune disease (e.g. scleroderma, Churg Strauss syndrome), celiac disease, drug reactions, and in association with the Hypereosinophilic syndrome. ${ }^{3}$ Atopy is the etiology in approximately $75 \%$ of the patients. ${ }^{5}$ Most of the patients are sensitized to environmental allergens and up to $62 \%$ of cases are sensitized to food. ${ }^{8}$

Primary is idiopathic and secondary is due to infections or connective tissue disorders where is the cause of eosinophilic aggregation can be ascertained.

The clinical features and the imaging findings depend on the segment of the bowel and layers of bowel involved. The disease can involve any segment of the bowel. Stomach and small intestine are the commonest sites of involvement. ${ }^{2}$ The pathological changes are seen in accordance with the involvement of the layer of bowel wall.

Mucosal involvement is commonest which manifests as prominent mucosal folds, polyps and ulcers. Patients present with decreased appetite, early satiety, vomiting, gastric dysmotility, abdominal pain, malabsorption, diarrhea, occult blood in stools and on imaging there is thickening of the bowel wall.2, 9 When the muscularis mucosa is involved, patients present with obstructive symptoms with reduced peristalsis and on imaging, diffuse bowel wall thickening, areas of reduced distensibility, strictures, or intestinal obstruction may be seen. ${ }^{2}$

In our case, initially only the mucosa was involved, so patient presented with pain and loose stools. Later when the muscularis was also involved, he presented with obstructive symptoms. With serosal involvement, patient presents with distension of abdomen and on imaging there may be ascites, pleural effusion, omental thickening, lymphadenopathy, or clustering of small bowel loops. Laboratory investigations reveal eosinophilic ascites and peripheral eosinophilia.2, 10

The differential diagnosis in case of involvement of the small intestine and large intestine would be those due to eosinophilic disorders or non eosinophilic disorders (inflammatory bowel disease, infectious diseases like tuberculosis, connective tissue diseases, neoplastic diseases like lymphoma, adenocarcinoma and malabsorptive disorders), Drug-induced colitis (Nonsteroidal antiinflammatory drugs, Rifampicin, Clozapine, Tacrolimus, Gold, Allogenic bone marrow transplant). Others: Tolosa Hunt syndrome, vasculitis, Churg Strauss syndrome) and Acute radiation colitis. ${ }^{3}$

Eosinophilic colitis in infants is a benign, frequently food-related entity and dietary elimination of the aggressor often resolves the disorder within days. Adolescent or older patients require more aggressive medical management including: glucocorticoids, anti- histamines, leukotriene receptor antagonists as well as novel approaches employing biologics that target interleukin-5 (IL-5) and IgE. ${ }^{3}$

The treatment for the eosinophilic enterocolitis mainly is avoidance of the allergen either food or environmental allergens. Identification of the particular allergen and complete avoidance of 
allergic food has shown complete remission of the disease and the symptoms. However many times this is not feasible, then treatment with corticosteroids is the option.

Though it is a rare disorder, it is important to consider this disease entity in the differentials with the bowel wall thickening and chronic symptoms. However arriving at the diagnosis is made difficult due to non-specific symptoms and no specific criteria for diagnosis. As the treatment is entirely different from other disease entities and prognosis is generally favorable with appropriate treatment it is important not to ignore the possibility of this disease entity.

The value of CT in eosinophilic enterocolitis does not lie only in its ability to rule out complications but also to demonstrate the distribution of the disease, to delineate accurately those bowel segments that show the most pronounced involvement and sometimes even to localize those layers within the affected bowel walls that show the most pronounced inflammation.

Especially if the typical mucosal and sub mucosal involvement is lacking, CT may help to decide whether to take the full thickness biopsy in order to avoid a repeated non-diagnostic endoscopic approach to this rare but principally benign condition, which usually shows a good response to corticosteroid therapy. CT also helps to assess changes after treatment, and the role of imaging in evaluating response to therapy continues to evolve and expand.

\section{BIBLIOGRAPHY:}

1. W. Wiesner, Th. Kocher, M. Heim et al. CT findings in eosinophilic enterocolitis with predominantly serosal and muscular bowel wall infiltration. JBR-BTR, 2002; 85: 4-6.

2. Alampady Krishna Prasad Shanbhogue, Srinivasa R. Prasad, Jaishree Jagirdar et al. Comprehensive update on select immune-mediated gastroenterocolitis syndromes: implications for diagnosis and management. Radiographics 2010; 30: 1465-87.

3. Abdul rahman A. Alfadda, Martin A. Storr, Eldon A. Shaffer et al. Eosinophilic colitis: epidemiology, clinical features, and current management. Ther Adv Gastroenterol 2010; 4 (5): $301-309$.

4. Kaijser R. Zur Kenntnis der allergischen affektionen des verdauungskanals vom standput des chirurgen aus. Arch Klin Chir 1937; 188: 36-64.)

5. Rothenberg ME. Eosinophilic gastrointestinal disorders. J Allergy Clin Immunol 2004; 113: 11 28.

6. Klein NC, Hargrove RL, Sleisenger MH, et al. Eosinophilic gastroenteritis. Medicine 1970; 49(4): 299 - 319.

7. Mueller S. Classification of eosinophilic gastrointestinal diseases. Best Pract Res Clin Gastroenterol 2008; 22: 425 - 40.

8. Guajardo JR, Plotnick LM, Fende JM, Collins MH, Putnam PE, Rothenberg ME. Eosinophilassociated gastrointestinal disorders: a world-wide-web based registry. J Pediatr 2002; 141: $576-81$.

9. David M. Fleischer, Dan Atkins et al. Evaluation of the Patient with Suspected Eosinophilic Gastrointestinal Disease. Immunol Allergy Clin N Am 2009; 29: 53 - 63.

10. Talley NJ, Shorter RG, Phillips SF, Zinsmeister AR. Eosinophilic gastroenteritis: a clinicopathological study of patients with disease of the mucosa, muscle layer, and subserosal tissues. Gut 1990; 31: 54 - 58. 


\section{CASE REPORT}

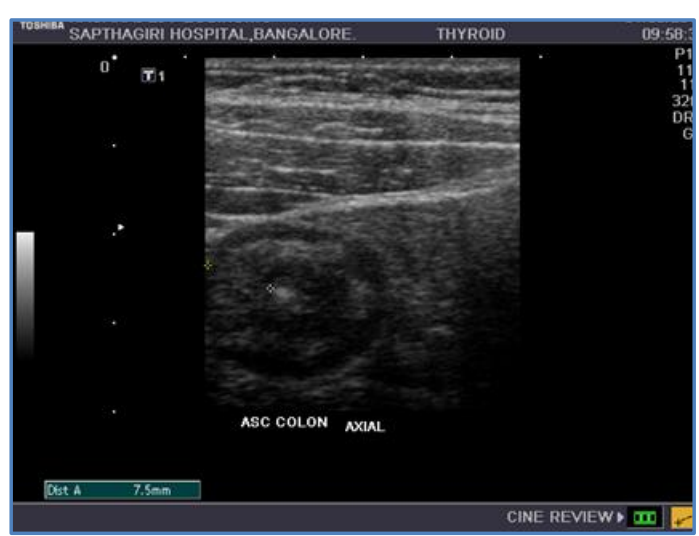

Fig. 1

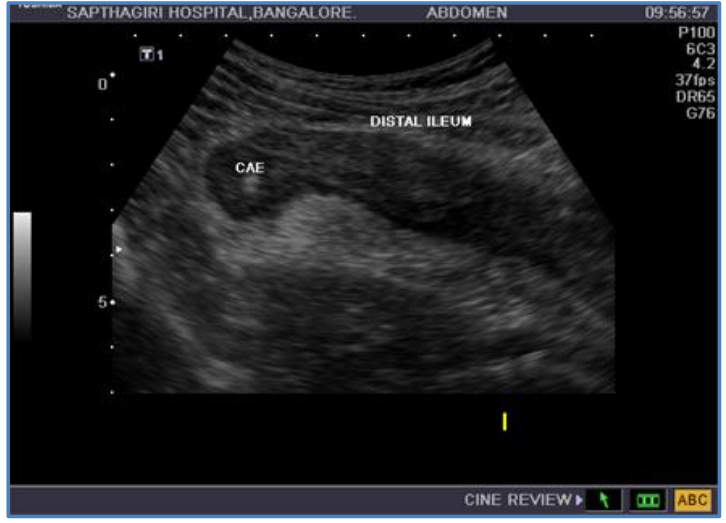

Fig. 2

Fig. 3: Trans abdominal ultrasound- Long segment diffuse circumferential wall thickening of distal ileum, caecum and adjacent ascending colon.

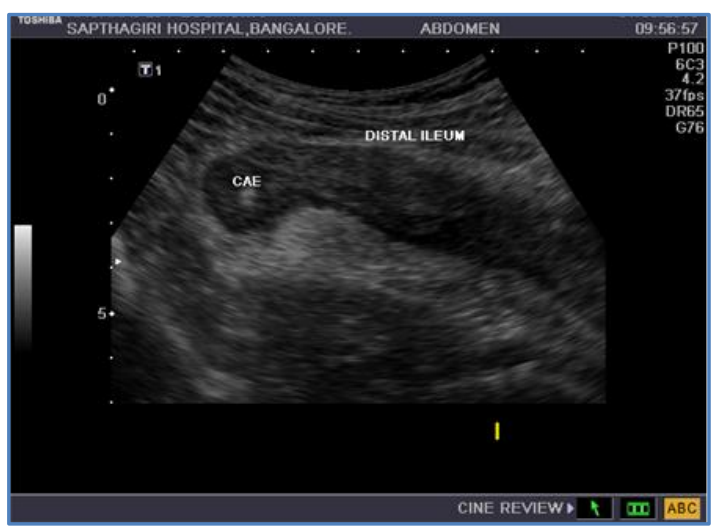

Fig. 3

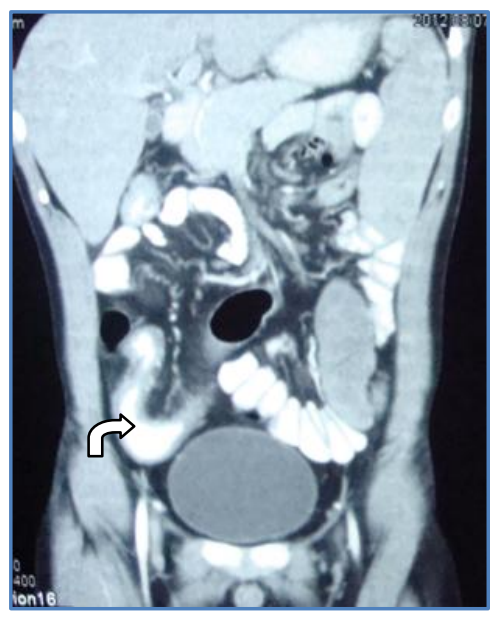

Fig. 4 


\section{CASE REPORT}

Fig. 5: Oral, rectal and IV contrast CT coronal and axial images showing enhancing diffuse wall thickening of the ileum, caecum and adjacent ascending colon.

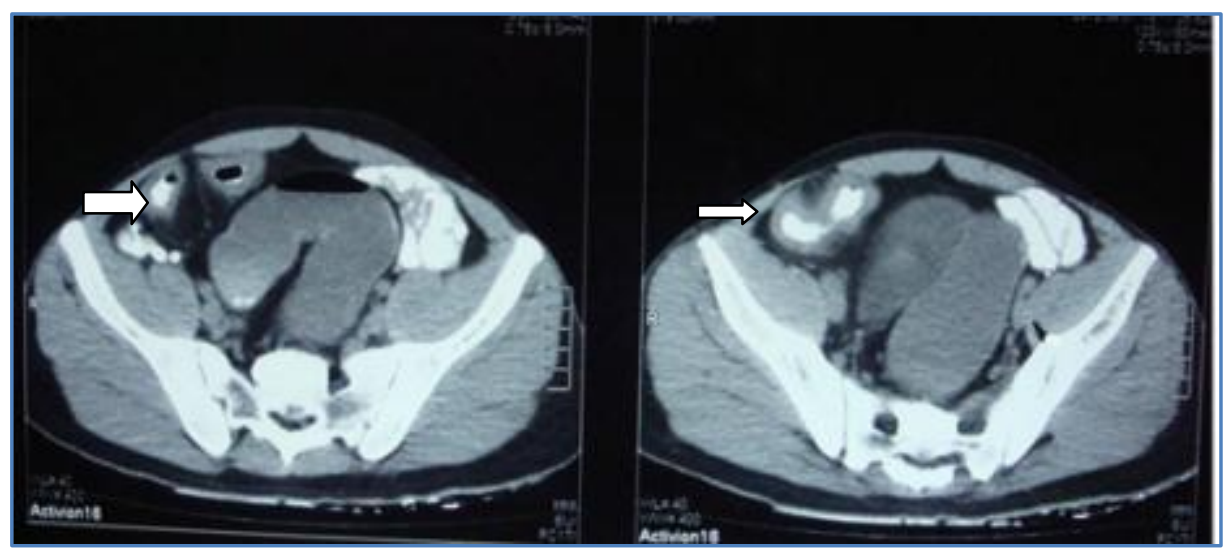

\section{Fig. 5}

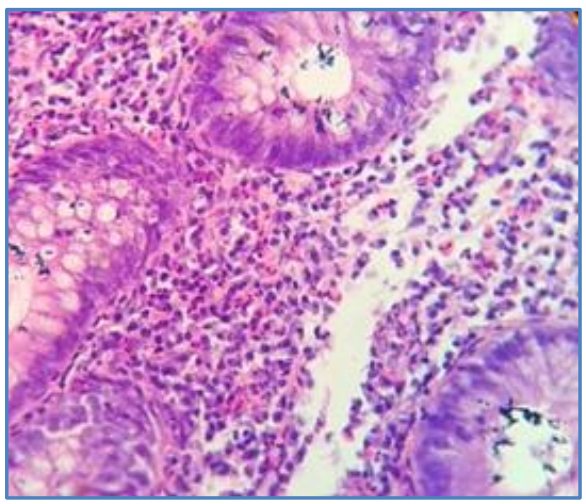

Fig. 6: Inflammation in the lamina propria with plenty of eosinophils

Fig. 7: Barium meal follows through examination - stricture at the terminal ileum with proximal small bowel loop dilation (fig 4).

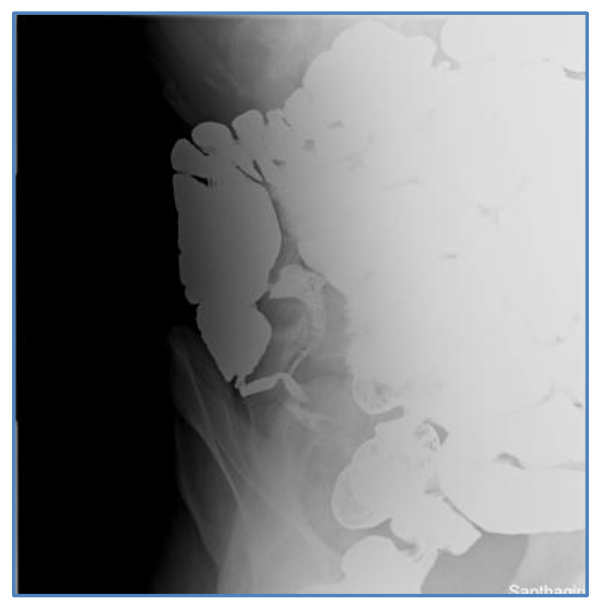

Fig. 7 


\section{CASE REPORT}

\section{AUTHORS:}

1. Nagarajaiah Y. N.

2. Prakash Jain R. B.

3. Nitash Prakash

\section{PARTICULARS OF CONTRIBUTORS:}

1. Assistant Professor, Department of Radiodiagnosis, Sapthagiri Medical College and Research Centre.

2. Assistant Professor, Department of Radiodiagnosis, Sapthagiri Medical College and Research Centre.

3. Radiologitst, Department of Radiodiagnosis, Sapthagiri Medical College and Research Centre.

\section{NAME ADDRESS EMAIL ID OF THE} CORRESPONDING AUTHOR:

Dr. Prakash Jain,

Department of Radiology,

Sapthagiri Medical College and

Research Centre,

Hesaraghatta Main Road,

Bangalore-560090.

Email: 2prakashjain@gmail.com

Date of Submission: 23/04/2014.

Date of Peer Review: 24/04/2014.

Date of Acceptance: 21/07/2014.

Date of Publishing: 24/07/2014. 